\title{
Medication Adherence and Persistence in Patients with Severe Major Depressive Disorder with Psychotic Features: Antidepressant and Second-Generation Antipsychotic Therapy Versus Antidepressant Monotherapy
}

\author{
Dawn N. Kim-Romo, PharmD, PhD; Karen L. Rascati, PhD; Kristin M. Richards, PhD; \\ Kentya C. Ford, DrPH; James P. Wilson, PhD; and Susan N. Beretvas, PhD
}

\begin{abstract}
BACKGROUND: Major depressive disorder with psychotic features, or psychotic depression, is a severe mental health disorder often associated with a worse depression-related symptom profile when compared with major depressive disorder without psychotic features. While combination pharmacotherapy with an antidepressant and an antipsychotic is recommended as first-line therapy, antidepressant monotherapy has been found to be useful and efficacious in psychotic depression.
\end{abstract}

OBJECTIVE: To assess the rates of antidepressant adherence and antidepressant persistence in Texas Medicaid patients with psychotic depression who used antidepressant plus second-generation antipsychotic (AD/SGA) therapy or antidepressant (AD) monotherapy.

METHODS: Using Texas Medicaid prescription and medical claims data from September 2007 to December 2012, adult patients aged 18-63 years were included if they had no confounding psychiatric disorders, no antidepressant claims during a 6-month pre-index period, and at least 1 diagnosis for severe major depressive disorder with psychotic features (ICD-9-CM codes 296.24 and 296.34). The first claim date for an antidepressant served as the index date. All patients were required to have at least 2 antidepressant claims, and those in the AD/SGA cohort were required to have 2 or more claims for an SGA. Study covariates included age, gender, race/ethnicity, residence, Charlson Comorbidity Index (CCI) score, and tobacco use/ dependence. Statistical analyses included descriptive statistics, univariate analyses, logistic regression, and Cox proportional hazards regression.

RESULTS: $A$ total of 926 patients met study criteria $(A D$ cohort $=510$; $A D / S G A$ cohort $=416)$. The overall sample had a mean $[ \pm S D]$ age of 40.5 [ \pm 13.2$]$ years and was primarily female $(66.8 \%)$ and non-Caucasian (74.8\%). When compared with the AD cohort, patients in the AD/SGA cohort had a $52.3 \%$ higher likelihood of being adherent to antidepressant therapy based on proportion of days covered (PDC; $0 \mathrm{R}=1.523 ; 95 \% \mathrm{Cl}=1.129$ 2.053; $P=0.006$ ). Similarly, antidepressant adherence was $42.0 \%$ higher for the $A D / S G A$ cohort based on medication possession ratio (MPR; $O R=1.420$; $95 \% \mathrm{Cl}=1.062-1.898 ; P=0.018$ ). Younger patients, African Americans, and tobacco users/dependents had significantly worse likelihoods of antidepressant medication adherence based on PDC and MPR. The risk of antidepressant nonpersistence was $23.2 \%$ lower for patients in the AD/SGA cohort ( $\mathrm{HR}=0.768 ; 95 \% \mathrm{Cl}=0.659-0.896 ; P=0.001$ ), compared with those in the $A D$ cohort. Antidepressant nonpersistence was significantly higher in younger patients, African Americans, Hispanics, and tobacco users/dependents.
CONCLUSIONS: Better antidepressant adherence and persistence outcomes were associated with combination pharmacotherapy with an $A D$ and an SGA antipsychotic. This study provides real-world estimates that support the current first-line treatment recommendations for psychotic depression; however, it should be noted that the majority of study patients used AD therapy only. Future research in psychotic depression is needed.

J Manag Care Spec Pharm. 2016;22(5):588-96

Copyright $\odot 2016$, Academy of Managed Care Pharmacy. All rights reserved.

\section{What is already known about this subject}

Previous research has reported worse health-related outcomes in psychotic depression when compared with major depressive disorder without psychotic features.

While an antidepressant plus an antipsychotic agent is advocated as first-line therapy by the American Psychiatric Association and the Texas Medication Algorithm Project practice guidelines, other therapy such as antidepressant monotherapy has been shown to be effective in the treatment of major depressive disorder with psychotic features.

Little is known about antidepressant adherence in major depressive disorder with psychotic features; however, adherence in patients with major depressive disorder is generally low.

\section{What this study adds}

This study provides estimates of proportion of days covered, medication possession ratio, and persistence in patients with major depressive disorder with psychotic features.

Study results found that patients on combination pharmacotherapy with an antidepressant plus a second-generation antipsychotic had significantly better antidepressant adherence and persistence compared with those on antidepressant monotherapy. Compared with Caucasians, African Americans had worse antidepressant adherence and persistence outcomes, whereas Hispanics had poorer rates of antidepressant persistence. 
$\mathrm{M}$ ajor depressive disorder with psychotic features is a severe subtype of major depressive disorder characterized as major depressive episodes with delusions, hallucinations, or both. ${ }^{1}$ This disorder, also termed psychotic depression, is associated with worse outcomes related to depressive episodes, suicide, hospitalizations, financial assistance, and social functioning when compared with patients with major depressive disorder without psychotic features. ${ }^{2-4}$ Epidemiological studies have estimated the prevalence of psychotic depression to be as low as $0.4 \%-0.6 \%$ in general populations and as high as $25.3 \%-44.7 \%$ in populations of patients with major depressive disorder. ${ }^{2,5-7}$ The financial burden of psychotic depression is apparent- 1 study from the United Kingdom reported inpatient costs for first-episode psychotic depression that were nearly equal to the inpatient costs associated with first-episode schizophrenia at estimates of $£ 2.6$ million and $£ 2.8$ million, respectively. ${ }^{8}$

Treatment goals for psychotic depression include depressive episode remission, attainment of baseline psychosocial functioning, and prevention of depression relapse and recurrence. ${ }^{9,10}$ While algorithms for psychotic depression exist, there is a lack of consensus among international practice guidelines. Generally, the American Psychiatric Association and the Texas Medication Algorithm Project guidelines recommend the use of combination therapy with an antidepressant and antipsychotic as first-line therapy, while the National Institute for Health and Clinical Excellence, the Danish Board of Health, and the Dutch National Steering Committee of Health guidelines support the use of antidepressant monotherapy. ${ }^{9-13}$ In general, the Texas Medication Algorithm Project guidelines recommend using an antipsychotic during the acute phase to achieve remission of symptoms and then continuing use for 1 to 2 months before slowly tapering off the antipsychotic, while antidepressants should be continued after symptom remission for 6 to 9 months at the full therapeutic doses used during the acute phase. ${ }^{10}$

A number of studies have found combination therapy with antidepressants plus second-generation antipsychotics (e.g., olanzapine, quetiapine, aripiprazole, and risperidone) to be effective in achieving a response or remission of depressive episode symptoms in psychotic depression. ${ }^{14-21}$ Combination therapy is more beneficial when compared with antipsychotic monotherapy, ${ }^{16,21}$ while the results are not as clear when combination therapy is directly compared with antidepressant monotherapy. ${ }^{14,15,22}$ For example, a meta-analysis found that combination therapy with an antidepressant plus antipsychotic was superior to antidepressant monotherapy in relation to study-defined inefficacy and Clinical Global Impressions scores. ${ }^{23}$ However, superiority was not found in depression ratings and psychosis ratings. Similarly, a Cochrane Review found that combination therapy was not necessarily more effective than antidepressant monotherapy. ${ }^{24}$ This is noteworthy, since antidepressant monotherapy of imipramine, nortriptyline, sertraline, or fluoxetine has been reported to be useful in treating psychotic depression..$^{14,22,25,26}$ Furthermore, antidepressant monotherapy and antidepressant/antipsychotic therapy have successfully treated acute depressive episode and have been shown to be efficacious in the long term. ${ }^{15}$

Previous related studies have primarily compared psychotically depressed patients with those with nonpsychotic depression. While these studies have reported outcomes associated with depression scores, relapse rates, psychosocial impairment, hospitalizations, length of stay, and outpatient visits, none have compared medication treatment regimens among patients with psychotic depression. ${ }^{2,4,27}$ Similarly, there is a lack of medication adherence and persistence studies focusing on psychotic depression. One study on psychotic depression evaluated medication use as a percentage of time and found that, at 24 months, $31.4 \%$ of patients continued to use their antidepressant medications $75 \%-100 \%$ of the time..$^{28}$ In general, patients with major depressive disorder have low antidepressant adherence and high rates of antidepressant discontinuation. ${ }^{29,30}$ One study reported an antidepressant adherence rate of $43.3 \%$ based on medication possession ratio, ${ }^{31}$ and another estimated antidepressant persistence rates of $43.7 \%-45.2 \%$. ${ }^{29}$ While these studies provide insight into antidepressant adherence and persistence in patients with major depressive disorder, the effect of psychotic features cannot be clearly delineated. ${ }^{29-31}$ Therefore, in order to provide more insight into the real-world treatment of psychotic depression, the objective of this study was to compare medication adherence and persistence among Texas Medicaid patients using combination pharmacotherapy with an antidepressant plus second-generation antipsychotic versus those on antidepressant therapy alone.

\section{Methods}

\section{Data Source}

A retrospective cohort design was employed using Texas Medicaid prescription and medical claims data from September 1, 2007, to December 31, 2012. Patients with a diagnosis of severe major depressive disorder with psychotic features were included if they had a 6-month pre-index period with no antidepressant or antipsychotic medication claims and a 12-month post-index period where they received antidepressant therapy. The first antidepressant claim date served as the index date, where possible index dates (i.e., the index period) ranged from as early as March 1, 2008, to as late as December 31, 2011, to allow for the minimum 18-month study period (Figure 1). All claims data were in de-identified form, and the study protocol was deemed as nonhuman subjects research and approved by The University of Texas at Austin Institutional Review Board.

\section{Inclusion and Exclusion Criteria}

Texas Medicaid patients with a diagnosis of severe major depressive disorder with psychotic features based on International Classification of Diseases, Ninth Revision, Clinical Modification 


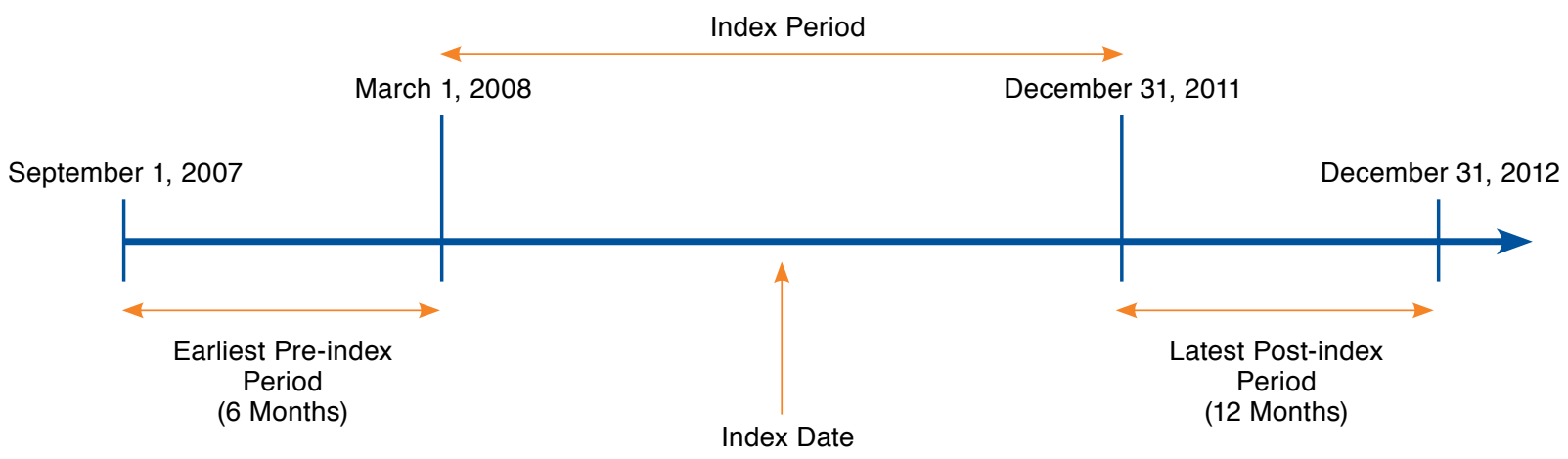

(ICD-9-CM) codes 296.24 and 296.34 were included if they met the following criteria: (a) were adults aged 18 to 63 years at index; (b) had at least 2 prescription claims for an antidepressant (AD cohort), or had at least 2 claims for an antidepressant and at least 2 claims for an oral or injectable second-generation antipsychotic agent (AD/SGA cohort); and (c) were continuously enrolled for 18 months (6-month baseline pre-index period and 12-month follow-up post-index period) in Texas Medicaid.

For the purpose of controlling for disease-related confounders, patients were excluded if they had an ICD-9-CM diagnosis of dementia (290.xx); schizophrenia (295.xx); pervasive development disorder (299.xx); mental retardation(317.xx-319.xx); other cerebral degenerations (331.xx); Parkinson's disease (332.xx); senility without mention of psychosis (797.xx); or manic depression, bipolar disorder, or cyclothymic disorder (296.0, 296.1, 296.4, 296.5, 296.6, 296.7, 296.80, 296.81, 296.89, and 301.13). Patients were excluded if they had a claim at any time for clozapine, a first-generation antipsychotic agent, or electroconvulsive therapy based on ICD-9-CM codes 94.26 and 94.27 or procedural codes 90870 and 90871 . Patients with SGA antipsychotic use during the pre-index period were also excluded. Patients were not included in the AD/SGA cohort if their first claim for an SGA did not occur on the index date or if there was less than a 30-day overlap of combined pharmacotherapy usage between the AD therapy and SGA therapy.

\section{Study Outcomes}

$\mathrm{AD}$ adherence was the study outcome of interest and included the following: (a) AD adherence based on proportion of days covered (PDC) $\geq 80 \%$ (defined as the number of days with medication available divided by 365 days); (b) $\mathrm{AD}$ adherence based on medication possession ratio (MPR) $\geq 80 \%$ (calculated as the sum of the days supply divided by 365 days); and (c) time until $\mathrm{AD}$ nonpersistence (i.e., the time, in days, before a 45-day gap in therapy occurred), with sensitivity analyses using 30-day and 60-day permissible gaps.

\section{Study Covariates}

The patient cohorts, $\mathrm{AD}$ versus AD/SGA, were the primary independent variables. Multivariate analyses controlled for the following demographic and clinical variables: age, gender, race/ ethnicity, place of residence, pre-index Charlson Comorbidity Index (CCI) score (Dartmouth-Mannitoba ${ }^{32}$ ), and post-index tobacco use/dependence based on ICD-9-CM codes 305.1, V15.82, 989.84, and 649.00 through 649.04 .

\section{Statistical Analyses}

Statistical analyses were performed using PASW Statistics 18 (formally SPSS Statistics, Chicago IL). All statistical analyses were two-tailed and utilized an a priori significance level of $P<0.05$. Descriptive statistics (means, standard deviations [SD], and frequencies) were calculated for all variables in the study. Other statistical analyses included Pearson's chi-square test for dichotomous variables, independent samples t-tests for normally distributed continuous variables, and Mann-Whitney $\mathrm{U}$ tests for nonparametric data. Multiple logistic regression analyses were used to assess the medication adherence variables of PDC and MPR. Time until AD nonpersistence was compared using Cox proportional hazards regression.

\section{Results}

A total of 926 patients met study criteria, with 416 and 510 patients in the AD/SGA and AD cohorts, respectively (Figure 2). Baseline and post-index characteristics for the total sample and individual cohorts are displayed in Table 1. At baseline, the AD/SGA cohort was, on average, significantly older and had a lower number of comorbidities based on CCI scores when compared with the AD cohort. Also, the AD/SGA cohort had a significantly lower percentage of females, Caucasians, and rural dwellers. There was no difference between the 2 cohorts regarding post-index tobacco use/dependence. The mean $[ \pm S D]$ SGA persistence rate was $178.6[ \pm 127.9]$ days, where $49.3 \%$ of the AD/SGA cohort utilized SGA therapy for 5 months or longer. 
Medication Adherence and Persistence in Patients with Severe Major Depressive Disorder with Psychotic Features: Antidepressant and Second-Generation Antipsychotic Therapy Versus Antidepressant Monotherapy

\section{FIGURE 2 Patient Selection in Texas Medicaid Database}

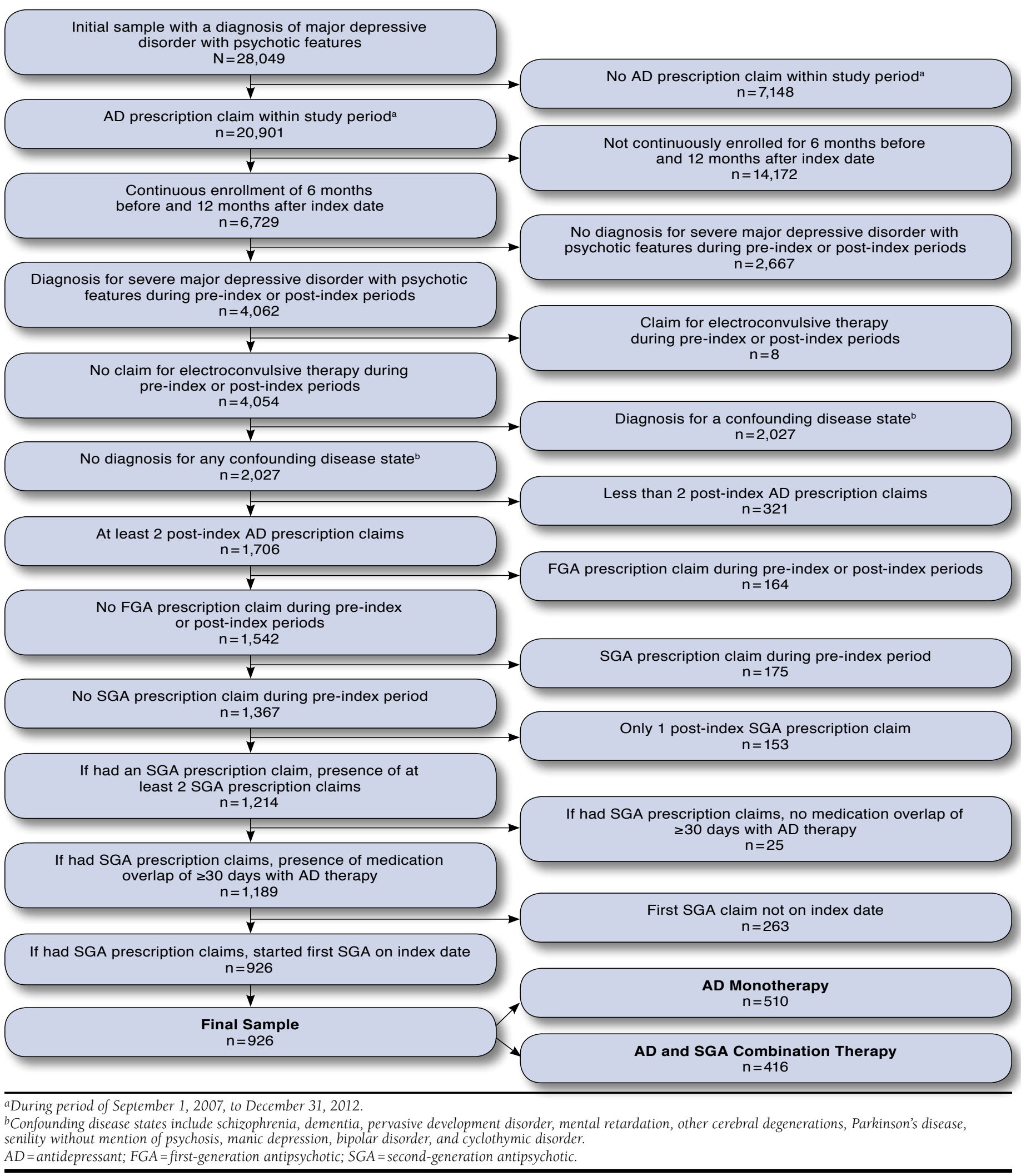




\section{Medication Adherence and Persistence in Patients with Severe Major Depressive Disorder with Psychotic Features: Antidepressant and Second-Generation Antipsychotic Therapy Versus Antidepressant Monotherapy}

TABLE 1 Sociodemographic and Clinical Characteristics

\begin{tabular}{|c|c|c|c|c|}
\hline & $\begin{array}{c}\text { Total } \\
(\mathrm{N}=926)\end{array}$ & $\begin{array}{c}\text { AD } \\
(n=510)\end{array}$ & $\begin{array}{l}\text { AD/SGA } \\
(n=416)\end{array}$ & $P$ Value $^{\mathrm{a}}$ \\
\hline Age, mean $[ \pm S D]^{b, c}$ & $40.5[ \pm 13.2]$ & $39.2[ \pm 13.3]$ & $42.0[ \pm 13.0]$ & 0.001 \\
\hline Gender (\%)d & & & & $<0.001$ \\
\hline Female & 66.8 & 72.2 & 60.3 & \\
\hline Male & 33.2 & 27.8 & 39.7 & \\
\hline Race/ethnicity (\%)d & & & & $<0.001$ \\
\hline Caucasian & 25.2 & 30.0 & 19.2 & \\
\hline African American & 34.9 & 28.0 & 43.3 & \\
\hline Hispanic & 36.7 & 39.2 & 33.7 & \\
\hline Othere & 3.2 & 2.7 & 3.8 & \\
\hline Residence (\%)d & & & & $<0.001$ \\
\hline Rural & 20.5 & 25.7 & 14.2 & \\
\hline Urban & 79.5 & 74.3 & 85.8 & \\
\hline $\mathrm{CCI}$, mean $[ \pm \mathrm{SD}]^{\mathrm{f}, \mathrm{g}}$ & $0.6 \quad[ \pm 1.3]$ & $0.8 \quad[ \pm 1.4]$ & $0.5 \quad[ \pm 1.2]$ & $<0.001$ \\
\hline Tobacco use/dependence (\%)d,h & 19.7 & 21.8 & 17.1 & 0.074 \\
\hline
\end{tabular}

a Significance at $P<0.05$.

bIndependent samples t-test.

cRepresents age, in years, at the time of first AD prescription claim.

dPearson's chi-square test.

'Other includes Asian, unknown.

fMann-Whitney U test.

gWeighted index based on the Dartmouth-Mannitoba adaptation.

hepresents the only post-index characteristic.

$A D=$ antidepressant $; C C I=$ Charlson Comorbidity Index; $S D=$ standard deviation; $S G A=$ second-generation antipsychotic

Approximately $33.0 \%$ and $38.3 \%$ of the total sample were adherent to their antidepressant medication therapy based on PDC and MPR, respectively. Specifically, the AD/SGA cohort had higher antidepressant adherence rates based on PDC (38.7\% vs. $28.4 \%$ ) and MPR (43.5\% vs. $34.1 \%$ ), compared with the AD cohort. A multivariate logistic regression analysis showed patients who used AD/SGA therapy had a 52.3\% significantly higher likelihood of being adherent to AD therapy based on PDC (odds ratio [OR] $=1.523 ; 95 \%$ confidence interval $[C I]=1.129-2.053 ; P=0.006$ ) versus the $A D$ cohort (Table 2). Similarly for MPR, the likelihood of AD adherence was significantly higher by approximately $42.0 \%$ in the AD/SGA cohort $(\mathrm{OR}=1.420 ; 95 \% \mathrm{CI}=1.062-1.898 ; P=0.018)$, while controlling for other variables in the model (Table 3). In both regression models, younger age, African-American race, and tobacco use/dependence were associated with lower likelihoods of AD adherence $(P<0.05)$.

The mean $[ \pm \mathrm{SD}]$ time until AD nonpersistence was 172.3 [ \pm 130.4$]$ days for the total sample. The AD persistence rate was, on average, significantly longer in the AD/SGA cohort than in the AD cohort by approximately 34 days (191.3 [ \pm 132.1$]$ vs. $156.8[ \pm 127.1]$ days). A Cox proportional hazards regression model found that the risk of AD nonpersistence was 23.2\% significantly lower in the AD/SGA cohort, compared with the $\mathrm{AD}$ cohort (hazard ratio $[\mathrm{HR}]=0.768 ; 95 \% \mathrm{CI}=0.659-0.896$;
$P=0.001)$. Younger age, African-American race, Hispanic ethnicity, and tobacco use/dependence were associated with significantly higher risks of AD nonpersistence (Table 4). Sensitivity analyses showed comparable results favoring the AD/SGA cohort when using a 30-day permissible gap (158.9 $[ \pm 126.6]$ vs. $130.7[ \pm 115.4] ; \mathrm{HR}=0.782 ; 95 \% \mathrm{CI}=0.670-0.913 ; P=0.002)$ and a 60-day permissible gap (216.2 [ \pm 133.9$]$ vs. $174.8[ \pm 130.9]$ days; $\mathrm{HR}=0.719 ; 95 \% \mathrm{CI}=0.611-0.840 ; P<0.001)$.

\section{Discussion}

This study assessed medication adherence and persistence among 2 cohorts (AD/SGA vs. AD) of Texas Medicaid patients who were diagnosed with severe major depressive disorder with psychotic features. Somewhat unexpectedly, more than half of the total sample used AD monotherapy $(n=510)$ compared with AD/SGA therapy $(n=416)$. This deviation from first-line recommendations could be explained by a number of reasons, which this study was unable to measure. Since it is standard practice for providers to weigh the risks versus benefits of treatment when prescribing therapy, ${ }^{10}$ present comorbidities (e.g., diabetes mellitus) and the known adverse events of antipsychotics, such as weight gain and increased risks of elevated blood glucose and lipids, could potentially influence prescribing habits favoring AD monotherapy. This may be the case in this study, since patients on AD monotherapy had a significantly higher mean CCI score when compared with patients 


\section{Medication Adherence and Persistence in Patients with Severe Major Depressive Disorder with Psychotic Features: Antidepressant and Second-Generation Antipsychotic Therapy Versus Antidepressant Monotherapy}

TABLE 2 Logistic Regression Analysis Comparing Likelihood of Antidepressant Adherence Based on PDC $\geq 80 \%$ Among Cohorts $(\mathrm{N}=926)$

\begin{tabular}{|c|c|c|c|c|c|}
\hline & OR & \multicolumn{2}{|c|}{$95 \%$ CI } & Wald $\chi^{2}$ & $P$ Value ${ }^{a}$ \\
\hline AD/SGA vs. ADb & 1.523 & 1.129 & 2.053 & 7.606 & 0.006 \\
\hline $\mathrm{Agec}^{\mathrm{c}}$ & 1.039 & 1.027 & 1.052 & 39.506 & $<0.001$ \\
\hline Female vs. male & 0.827 & 0.610 & 1.121 & 1.494 & 0.222 \\
\hline African American vs. Caucasian & 0.612 & 0.419 & 0.894 & 6.462 & 0.011 \\
\hline Hispanic vs. Caucasian & 0.744 & 0.505 & 1.096 & 2.242 & 0.134 \\
\hline Otherd vs. Caucasian & 1.232 & 0.552 & 2.748 & 0.259 & 0.611 \\
\hline Urban vs. rural & 0.738 & 0.509 & 1.069 & 2.580 & 0.108 \\
\hline $\mathrm{CCI}^{\mathrm{e}}$ & 0.919 & 0.817 & 1.034 & 1.957 & 0.162 \\
\hline Tobacco use/dependence vs. none $\mathrm{f}^{\mathrm{f}}$ & 0.636 & 0.437 & 0.927 & 5.546 & 0.019 \\
\hline
\end{tabular}

a Significance at $P<0.05$.

${ }^{b}$ Adherent $(P D C \geq 80 \%)$ : AD/SGA cohort $=38.7 \%$; AD cohort $=28.4 \%$.

CRepresents age, in years, at the time of first AD prescription claim.

${ }^{d} O$ ther includes Asian, unknown.

'Weighted index based on the Dartmouth-Mannitoba adaptation.

fRepresents the only post-index characteristic.

$A D=$ antidepressant; $C C I=$ Charlson Comorbidity Index; $C I=$ confidence interval; $O R=$ odds ratio; $P D C=$ proportion of days covered; $S G A=$ second-generation antipsychotic.

\section{TABLE 3 Logistic Regression Analysis Comparing Likelihood of Antidepressant Adherence} Based on MPR $\geq 80 \%$ Among Cohorts ( $N=926$ )

\begin{tabular}{|c|c|c|c|c|c|}
\hline & OR & \multicolumn{2}{|c|}{$95 \%$ CI } & Wald $\chi^{2}$ & $P$ Value $^{\mathrm{a}}$ \\
\hline AD/SGA vs. ADb & 1.420 & 1.062 & 1.898 & 5.596 & 0.018 \\
\hline Age $^{\mathrm{C}}$ & 1.044 & 1.031 & 1.056 & 51.360 & $<0.001$ \\
\hline Female vs. male & 0.922 & 0.684 & 1.242 & 0.285 & 0.594 \\
\hline African American vs. Caucasian & 0.649 & 0.448 & 0.938 & 5.290 & 0.021 \\
\hline Hispanic vs. Caucasian & 0.800 & 0.548 & 1.168 & 1.336 & 0.248 \\
\hline Otherd vs. Caucasian & 1.363 & 0.607 & 3.060 & 0.564 & 0.453 \\
\hline Urban vs. rural & 0.758 & 0.528 & 1.089 & 2.249 & 0.134 \\
\hline $\mathrm{CCI}^{\mathrm{e}}$ & 0.940 & 0.841 & 1.051 & 1.183 & 0.277 \\
\hline Tobacco use/dependence vs. nonef & 0.669 & 0.467 & 0.959 & 4.788 & 0.029 \\
\hline
\end{tabular}

a Significance at $P<0.05$.

${ }^{b}$ Adherent $(M P R \geq 80 \%)$ : AD/SGA cohort $=43.5 \%$; AD cohort $=34.1 \%$.

cRepresents age, in years, at the time of first AD prescription claim.

dOther includes Asian, unknown.

'Weighted index based on the Dartmouth-Mannitoba adaptation.

fRepresents the only post-index characteristic.

$A D=$ antidepressant; $C C I=$ Charlson Comorbidity Index; $C I=$ confidence interval; $M P R=$ medication possession ratio; $O R=$ odds ratio; $S G A=$ second-generation antipsychotic.

on AD/SGA therapy. Therefore, it is a possibility that patients deemed to have "less severe" psychotic depression were initiated with $\mathrm{AD}$ monotherapy after weighing the risks versus benefits of dual therapy.

To our knowledge, medication adherence measures using MPR and PDC have not been previously used in evaluating treatment adherence in psychotic depression. Craig et al. (2007) analyzed AD usage based on patient interviews, ${ }^{28}$ but the present study represents the first known rates of $A D$ adherence in psychotic depression based on PDC and MPR. Similarly, there are no known studies that evaluate medication persistence in severe major depressive disorder with psychotic features. The authors support that the results of this study can help offer insight into practice guideline adherence among providers. For example, the Texas Medication Algorithm Project guidelines recommend using an antipsychotic medication for 1 to 2 months after a clinical response is achieved, ${ }^{10}$ and Wijkstra et al. (2010) support the continued use of an SGA for 4 months after a successful 2-month acute treatment course..$^{15}$ These recommendations are comparable with the result of this study in which SGA therapy was continuously used for approximately 6 months before a treatment gap occurred. Conversely, our results show that patients persisted on their AD medications for less than 6 months, which is less than the recommended 


\section{Medication Adherence and Persistence in Patients with Severe Major Depressive Disorder with Psychotic Features: Antidepressant and Second-Generation Antipsychotic Therapy Versus Antidepressant Monotherapy}

TABLE 4 Cox Proportional Hazards Regression Model Comparing Time to Nonpersistence (Based on 45-Day Permissible Gap of No Antidepressant Medication) Among Cohorts ( $\mathrm{N}=926$ )

\begin{tabular}{|c|c|c|c|c|c|}
\hline & HR & \multicolumn{2}{|c|}{$95 \%$ CI } & Wald $\chi^{2}$ & $P$ Value $^{\mathrm{a}}$ \\
\hline AD/SGA vs. AD ${ }^{b}$ & 0.768 & 0.659 & 0.896 & 11.283 & 0.001 \\
\hline $\mathrm{Age}^{\mathrm{c}}$ & 0.982 & 0.976 & 0.988 & 36.684 & $<0.001$ \\
\hline Female vs. male & 1.025 & 0.876 & 1.200 & 0.097 & 0.755 \\
\hline African American vs. Caucasian & 1.455 & 1.192 & 1.776 & 13.613 & $<0.001$ \\
\hline Hispanic vs. Caucasian & 1.301 & 1.061 & 1.594 & 6.408 & 0.011 \\
\hline Otherd vs. Caucasian & 1.204 & 0.783 & 1.852 & 0.713 & 0.398 \\
\hline Urban vs. rural & 1.133 & 0.934 & 1.375 & 1.610 & 0.205 \\
\hline $\mathrm{CCI}^{\mathrm{e}}$ & 1.010 & 0.956 & 1.067 & 0.124 & 0.725 \\
\hline Tobacco use/dependence vs. nonef & 1.249 & 1.040 & 1.501 & 5.678 & 0.017 \\
\hline
\end{tabular}

a Significance at $P<0.05$.

${ }^{b}$ Mean $[ \pm S D]$ antidepressant persistence, in days, based on a 45-day permissible gap: AD/SGA cohort $=191.3$ [ \pm 312.1$] ; A D$ cohort $=156.8$ [ \pm 127.1$]$.

cRepresents age, in years, at the time of first AD prescription claim.

${ }^{d}$ Other includes Asian, unknown.

'Weighted index based on the Dartmouth-Mannitoba adaptation.

fRepresents the only post-index characteristic.

$A D=$ antidepressant; $C C I=$ Charlson Comorbidity Index; $C I=$ confidence interval; $H R=$ hazard ratio; $S D=$ standard deviation; $S G A=$ second-generation antipsychotic

6-9 months of AD continuation treatment after the remission of symptoms occurs. ${ }^{10}$ However, the possibility that patients were appropriately titrated by their physicians once their symptoms improved cannot be ruled out.

Finally, this study provides a unique addition to the present literature, since there remains a dearth of information regarding minority and Medicaid-qualifying indigent populations with psychotic depression. African-American and Hispanic minorities had poorer AD adherence and/or persistence compared with Caucasians, which has also been supported in other depression studies. ${ }^{29,31}$ Additionally, this study offered more insight with regard to tobacco use/dependence-tobacco users and dependents had significantly worse rates of AD adherence (based on MPR and PDC) and AD persistence. Ultimately, the authors believe that further research on tobacco use and dependence may be necessary in order to better understand the treatment outcomes associated with psychotic depression.

\section{Limitations}

While this is the first known study to analyze psychotic depression in relation to PDC, MPR, and medication persistence, there are several limitations to consider while interpreting the study results. First, the data only represent prescription claims-they are not necessarily indicative of actual prescription usage. However, this limitation was partially reduced by including only the patients that had at least 2 prescription claims as a proxy for medication usage. Second, tobacco use/ dependence is likely to be underreported in large claims databases. Third, the study's generalizability is limited to similar populations-in this case, Medicaid-qualifying indigent populations with high rates of African Americans and Hispanics who were first-time users (or those with at least a 6-month wash-out period) of antidepressant and antipsychotic therapy for psychotic depression. Fourth, the authors cannot rule out coding inaccuracies associated with administrative claims data. Finally, since propensity scoring was not possible due to the already limited sample sizes and randomization was not an option, cohorts were significantly different at baseline, that is, patients in the AD cohort were younger and sicker based on CCI scores, which may have affected the overall adherence and persistence rates. However, in order to better control for this limitation, multivariate statistical analyses were employed.

Overall, selection bias was problematic in this study, since outcomes may have been due in part to inherent differences among patients instead of to the type of medication therapy received. Mental health scores and relapse rates were not available for this study, and as a result, the authors were unable to assess whether 1 cohort was more likely to consist of patients with a higher degree of depression or psychosis severity over the other. Similarly, the effect of physicianprescribing behavior and guideline adoption rates on study outcomes could not be assessed. It is possible that the lack of guideline adoption may have contributed to the larger sample of AD monotherapy users in this study ${ }^{33}$; although notably, the Texas Medication Algorithm Project guidelines, which advocate the use of combination pharmacotherapy as firstline, have been available since $1999 .{ }^{10}$ Finally, it is estimated that $36.6 \%-59.0 \%$ of patients with major depressive disorder smoke tobacco in order to self-relieve the psychological and physical symptoms of depression ${ }^{34}$; therefore, the lack of information on patient self-medication with tobacco may be an important limitation. 


\section{Medication Adherence and Persistence in Patients with Severe Major Depressive Disorder with Psychotic Features: Antidepressant and Second-Generation Antipsychotic Therapy Versus Antidepressant Monotherapy}

\section{Conclusions}

Overall, the results of this study indicate that users of combination pharmacotherapy with an AD and an SGA had significantly better AD adherence compared with those on AD therapy alone. Also, combination pharmacotherapy users fared significantly better with regard to AD persistence. Nonetheless, there were a high number of AD monotherapy users in this study, indicating a notable deviation from the first-line treatment recommendations recommended by U.S. practice guidelines for psychotic depression.

\section{Authors}

DAWN N. KIM-ROMO, PharmD, PhD; KAREN L. RASCATI, PhD; KENTYA C. FORD, DrPH; and JAMES P. WILSON, PhD, Health Outcomes \& Pharmacy Practice, College of Pharmacy, The University of Texas at Austin. KRISTIN M. RICHARDS, PhD, Center for Pharmacoeconomic Studies, College of Pharmacy, and SUSAN N. BERETVAS, PhD, Research and Graduate Studies, College of Education, The University of Texas at Austin.

AUTHOR CORRESPONDENCE: Karen L. Rascati, PhD, Professor of Health Outcomes \& Pharmacy Practice, The University of Texas at Austin College of Pharmacy, 1 University Station, A1900, Austin, TX 78712. Tel.: 512.471.1637; Fax: 512.471.8762;

E-mail:krascati@mail.utexas.edu.

\section{DISCLOSURES}

Kim-Romo received funding to conduct this study from the PhRMA Foundation Pre-Doctoral Fellowship in Health Outcomes. Rascati, Richards, Ford, Wilson, and Beretvas declare no conflict of interest in relation to this manuscript.

Kim-Romo and Rascati collaborated on the study design, data analysis, study interpretation, and writing of this manuscript. Richards, Ford, Wilson, and Beretvas provided critical evaluation of the study design, analysis, and interpretation, as well as edited this manuscript.

\section{REFERENCES}

1. American Psychiatric Association. Diagnostic and Statistical Manual of Mental Disorders. 4th ed. Text Revision. Washington, DC: American Psychiatric Association; 2000.

2. Johnson J, Horwath E, Weissman MM. The validity of major depression with psychotic features based on a community study. Arch Gen Psychiatry. 1991:48(12):1075-81

3. Gaudiano BA, Young D, Chelminski I, Zimmerman M. Depressive symptom profiles and severity patterns in outpatients with psychotic vs nonpsychotic major depression. Compr Psychiatry. 2008;49(5):421-29.

4. Gaudiano BA, Dalrymple KL, Zimmerman M. Prevalence and clinical characteristics of psychotic versus nonpsychotic major depression in a general psychiatric outpatient clinic. Depress Anxiety. 2009;26(1):54-64.

5. Ohayon MM, Schatzberg AF. Prevalence of depressive episodes with psychotic features in the general population. Am J Psychiatry. 2002;159(11): 1855-61.

6. Coryell W, Pfohl B, Zimmerman M. The clinical and neuroendocrine features of psychotic depression. J Nerv Ment Dis. 1984;172(9):521-28.
7. Meyers BS, Greenberg R. Late-life delusional depression. J Affect Disord. 1986;11(2):133-37.

8. Crebbin K, Mitford E, Paxton R, Turkington D. First-episode psychosis: an epidemiological survey comparing psychotic depression with schizophrenia. J Affect Disord. 2008;105(1-3):117-24

9. American Psychiatric Association. Practice guideline for the treatment of patients with major depressive disorder. Third edition. October 2010. Available at: https://psychiatryonline.org/pb/assets/raw/sitewide/practice_ guidelines/guidelines/mdd.pdf. Accessed March 27, 2016.

10. Crismon ML, Trivedi M, Pigott TA, et al. The Texas Medication Algorithm Project: report of the Texas Consensus Conference Panel on Medication Treatment of Major Depressive Disorder. J Clin Psychiatry. 1999;60(3):142-56.

11. National Collaborating Centre for Mental Health. Depression: The Treatment and Management of Depression in Adults. Updated ed. Leicester, UK: British Psychological Society; 2010. (NICE Clinical Guidelines, No. 90).

12. Danish Board of Health. Guidelines for the treatment of unipolar depression in adults. 2007. Available at: http://www.sst.dk/publ/Publ2007/PLAN/ SfR/SST_Dep.rapport.pdf. Accessed March 27, 2016.

13. Dutch National Steering Committee Multidisciplinary Guideline Development Mental Health. [Multidisciplinary guideline depression.] 2005. [In Dutch]. Available at: http://www.ggzrichtlijnen.nl/index.php?pagina=/ richtlijn/item/pagina.php\&richtlijn_id=43. Accessed April 3, 2016.

14. Wijkstra J, Burger H, van den Broek WW, et al. Treatment of unipolar psychotic depression: a randomized, double-blind study comparing imipramine, venlafaxine, and venlafaxine plus quetiapine. Acta Psychiatr Scand. 2010;121(3):190-200

15. Wijkstra J, Burger H, van den Broek WW, et al. Long-term response to successful acute pharmacological treatment of psychotic depression. J Affect Disord. 2010;123(1-3):238-42.

16. Meyers BS, Flint AJ, Rothschild AJ, et al. A double-blind randomized controlled trial of olanzapine plus sertraline vs olanzapine plus placebo for psychotic depression: the study of pharmacotherapy of psychotic depression (STOP-PD). Arch Gen Psychiatry. 2009;66(8):838-47.

17. Konstantinidis A, Hrubos W, Nirnberger G, et al. Quetiapine in combination with citalopram in patients with unipolar psychotic depression. Prog Neuropsychopharmacol Biol Psychiatry. 2007;31(1):242-47.

18. Gabriel A. Risperidone, quetiapine, and olanzapine adjunctive treatments in major depression with psychotic features: a comparative study. Neuropsychiatr Dis Treat. 2013;9:485-92.

19. Matthews JD, Siefert C, Dording C, et al. An open study of aripiprazole and escitalopram for psychotic major depressive disorder. J Clin Psychopharmacol. 2009;29(1):73-76.

20. Matthews JD, Bottonari KA, Polania LM, et al. An open study of olanzapine and fluoxetine for psychotic major depressive disorder: interim analyses. J Clin Psychiatry. 2002;63(12):1164-70

21. Rothschild AJ, Williamson DJ, Tohen MF, et al. A double-blind, randomized study of olanzapine and olanzapine/fluoxetine combination for major depression with psychotic features. J Clin Psychopharmacol. 2004;24(4):365-73.

22. Mulsant BH, Sweet RA, Rosen J, et al. A double-blind randomized comparison of nortriptyline plus perphenazine versus nortriptyline plus placebo in the treatment of psychotic depression in late life.J Clin Psychiatry. 2001;62(8):597-604

23. Farahani A, Correll CU. Are antipsychotics or antidepressants needed for psychotic depression? A systematic review and meta-analysis of trials comparing antidepressant or antipsychotic monotherapy with combination treatment. J Clin Psychiatry. 2012;73(4):486-96.

24. Wijkstra J, Lijmer J, Balk F, Geddes J, Nolen WA. Pharmacological treatment for psychotic depression. Cochrane Database Syst Rev. 2005(4): CD004044. 
25. Bruijn JA, Moleman P, Mulder PG, van den Broek WW. Treatment of mood-congruent psychotic depression with imipramine. J Affect Disord. 2001;66(2-3):165-74.

26. Zanardi R, Franchini L, Gasperini M, Perez J, Smeraldi E. Double-blind controlled trial of sertraline versus paroxetine in the treatment of delusional depression. Am J Psychiatry. 1996;153(12):1631-33.

27. Buoli M, Caldiroli A, Altamura AC. Psychotic versus non-psychotic major depressive disorder: a comparative naturalistic study. Asian J Psychiatr. 2013;6(4):333-37.

28. Craig TJ, Grossman S, Bromet EJ, Fochtmann LJ, Carlson GA. Medication use patterns and two-year outcome in first-admission patients with major depressive disorder with psychotic features. Compr Psychiatry. 2007;48(6):497-503.

29. Wu CH, Erickson SR, Piette JD, Balkrishnan R. The association of race, comorbid anxiety, and antidepressant adherence among Medicaid enrollees with major depressive disorder. Res Social Adm Pharm. 2012;8(3):193-205.
30. Olfson M, Marcus SC, Tedeschi M, Wan GJ. Continuity of antidepressant treatment for adults with depression in the United States.

Am J Psychiatry. 2006;163(1):101-08.

31. Cantrell CR, Eaddy MT, Shah MB, Regan TS, Sokol MC. Methods for evaluating patient adherence to antidepressant therapy: a real-world comparison of adherence and economic outcomes. Med Care. 2006;44(4):300-03.

32. Romano PS, Roos LL, Jollis JG. Adapting a clinical comorbidity index for use with ICD-9-CM administrative data: differing perspectives. J Clin Epidemiol. 1993;46(10):1075-79; discussion 1081-90.

33. Bauer MS. A review of quantitative studies of adherence to mental health clinical practice guidelines. Harv Rev Psychiatry. 2002;10(3):138-53.

34. Ziedonis D, Hitsman B, Beckham JC, et al. Tobacco use and cessation in psychiatric disorders: National Institute of Mental Health report. Nicotine Tob Res. 2008;10(12):1691-715. 